\title{
Critical Period Window for Spectral Tuning Defined in the Primary Auditory Cortex (A1) in the Rat
}

\author{
Etienne de Villers-Sidani, ${ }^{1}$ Edward F. Chang, ${ }^{1}$ Shaowen Bao, ${ }^{2}$ and Michael M. Merzenich ${ }^{1}$ \\ ${ }^{1}$ W. M. Keck Center for Integrative Neuroscience, Coleman Laboratory, Department of Otolaryngology, University of California, San Francisco, San \\ Francisco, California 94143, and ${ }^{2}$ Helen Wills Neuroscience Institute, University of California, Berkeley, Berkeley, California, 94720
}

\begin{abstract}
Experience-dependent plasticity during development results in the emergence of highly adapted representations of the external world in the adult brain. Previous studies have convincingly shown that the primary auditory cortex (A1) of the rat possesses a postnatal period of sensory input-driven plasticity but its precise timing (onset, duration, end) has not been defined. In the present study, we examined the effects of pure-tone exposure on the auditory cortex of developing rat pups at different postnatal ages with a high temporal resolution. We found that pure-tone exposure resulted in profound, persistent alterations in sound representations in A1 only if the exposure occurred during a brief period extending from postnatal day 11 (P11) to P13. We also found that postnatal sound exposure in this epoch led to striking alterations in the cortical representation of sound intensity.
\end{abstract}

Key words: plasticity; sensory; development; learning; environment; frequency

\section{Introduction}

It is generally recognized that early life experiences have a major, long-lasting impact on adult brain perceptions. Impacts are achieved through experience-dependent plasticity, which enables individuals to adapt to a wide range of environments that can hardly be predicted by their genetic makeup (Rauschecker, 1999). This brief postnatal epoch of heightened nervous system receptivity is commonly referred to as the critical period (CP) (Wiesel and Hubel, 1965; Simons and Land, 1987). Although lifelong learning is possible, neural plasticity during the CP is extreme, facilitating the adaptation of the developing brain to its environment and providing it with a stable long-lasting experiential foundation. The amazing ease with which infants raised in different cultures or by different parents can naturally acquire the language of their surroundings (Goodman and Nusbaum, 1994) is almost certainly a manifestation of this early, intense cerebral plasticity.

A time-limited period of brain plasticity in development can potentially be problematic, when associated with an impoverished or distorted early environment. Normal development of spectral tuning is greatly altered in infant kittens or rats exposed to tonal stimuli (Clements and Kelly, 1978; Zhang et al., 2001) and is grossly degraded when rats are reared through the CP in modulated or continuous noises (Bao et al., 2003a; Chang and Merzenich, 2003), and continuous noise exposure results in a

Received July 27, 2006; revised Dec. 1, 2006; accepted Dec. 2, 2006.

This work was supported by National Institutes of Health Grants NS-10414 and P02 NS34835-09, the Sandler Fund, and the Fonds de Recherche en Santé du Québec. We thank Tom Babcock for technical support and Rob Froemke, Dave Blake, Ram Ramachandran, and Dan Polley for their useful comments on this manuscript.

Correspondence should be addressed to Dr. Etienne de Villers-Sidani, W. M. Keck Center for Integrative Neuroscience, Coleman Laboratory, Department of Otolaryngology, Box 0732, University of California, San Francisco, 513 Parnassus Avenue, Room HSE-808, San Francisco, CA 94143. E-mail: etienne@phy.ucsf.edu.

D01:10.1523/JNEUROSCI.3227-06.2007

Copyright $\odot 2007$ Society for Neuroscience $\quad$ 0270-6474/07/270180-10\$15.00/0 prolongation of the CP duration. In humans, a history of chronic otitis media can affect the rate and quality of acquisition of normal language abilities (Moore et al., 1991). Infants raised with continuously closed Eustachian tubes attributable to a deep cleft palate similarly develop degraded language abilities and cognitive impairments and are failed readers, unless their cleft palate is surgically repaired at a young developmental age. With their inherited defect in place, all language inputs received by these infants are grossly degraded; with surgical correction, high-fidelity speech reception is restored (Dorf and Curtin, 1982; Richman et al., 1988).

The onset and duration of CP plasticity varies by sensory modality and in different mammalian or avian species. The CP is only a few days long and ends at around postnatal day 5 (P5) in the rat barrel cortex (Rice and Van der Loos, 1977; Schlaggar and O'Leary, 1993) although it extends for 2 weeks, beginning in the third postnatal week in the rat visual cortex (Fagiolini et al., 1994). Interestingly, in the visual system of several species, the onset of monocular deprivation-related plasticity is closely linked to the completion of visual acuity development (Berardi et al., 2000, 2003; Stern et al., 2001). Further supporting the concept that regulation of the $\mathrm{CP}$ window is tied to sensory experience, normally patterned inputs are necessary for the development of normal cortical columnar organization and the timing of CP offset (Fagiolini et al. 1994; Zhang et al., 2002; Iwai et al. 2003; Chang and Merzenich, 2003). This way, a premature closure of the CP could be prevented if insufficient or incoherent inputs are present during development.

Establishing the onset and duration of experience-dependent plasticity in the visual cortex has been instrumental for identifying many key aspects of its regulation (Taha and Stryker, 2005). Although the critical period in the auditory cortex is known to follow the onset of low-threshold hearing at P11-P12, and is known not to extend beyond the first month of postnatal life 
(Zhang et al., 2001), its onset and duration have not been determined in any mammalian species up to this time. As a first step toward a better understanding of the mechanisms regulating the $\mathrm{CP}$ in the auditory system, we here quantified the age-dependent effects of a monotone exposure on spectral tuning in the rat primary auditory cortex (A1). We found that our exposure protocol had profound persistent effects on frequency representation in the cortex only if the exposure started after P10 and before P14. We also found that this short $3 \mathrm{~d}$ period of plasticity coincides with an epoch of rapid maturation of cortical responses to simple tonal stimuli.

\section{Materials and Methods}

Mapping the auditory cortex. All procedures were approved under University of California San Francisco Animal Care Facility protocols. Seventy-two female Sprague Dawley rats aged P10 to P30 were premedicated with atropine sulfate $(0.02 \mathrm{mg} / \mathrm{kg})$ to minimize bronchial secretions and with dexamethasone $(0.2 \mathrm{mg} / \mathrm{kg})$ to minimize brain edema. They were then anesthetized with pentobarbital (35-60 mg/kg) and supplemental doses of dilute pentobarbital $(10 \mathrm{mg} / \mathrm{kg}$, i.p.) were given if necessary. Throughout the recording session, the depth of anesthesia was adjusted to keep the rat in an areflexic state while maintaining a physiological breathing rate. The cisterna magnum was drained of cerebrospinal fluid to minimize cerebral edema. The skull was secured in a head holder leaving the ears unobstructed. After, the right temporalis muscle was reflected, the auditory cortex was exposed and the dura was resected. The cortex was maintained under a thin layer of viscous silicone oil to prevent desiccation. Recording sites were marked on an amplified digital image of the cortical surface vasculature.

Cortical responses were recorded with tungsten microelectrodes (1-2 $\mathrm{M} \Omega$ at $1 \mathrm{kHz}$; FHC, Bowdoinham, ME). Recording sites were chosen to sample evenly from the auditory cortex at interelectrode distances of $125-175 \mathrm{~mm}$, carefully avoiding surface blood vessels. At every recording site, the microelectrode was lowered orthogonally into the cortex to a depth of 470-600 $\mu \mathrm{m}$ (layers 4/5), where vigorous stimulus-driven responses were obtained. The neural signal was amplified $(10,000 \times)$, filtered $(0.3-3 \mathrm{kHz})$, and monitored on-line. Acoustic stimuli were generated using TDT System III (Tucker-Davis Technology, Alachua, FL) and delivered to the left ear through a calibrated earphone (STAX 54) with a sound tube positioned inside the external auditory meatus. A software package (SigCal, SigGen, and Brainware; Tucker-Davis Technology) was used to calibrate the earphone, generate acoustic stimuli, monitor cortical response properties on-line, and store data for off-line analysis. The evoked spikes of a single neuron or a small cluster of neurons were collected at each site.

Frequency-intensity receptive fields (RFs) were reconstructed in detail by presenting pure tones of 50 frequencies $(1-30 \mathrm{kHz} ; 0.1$ octave increments; $25 \mathrm{~ms}$ duration; $5 \mathrm{~ms}$ ramps) at eight sound intensities [0-70 dB sound pressure level (SPL) in $10 \mathrm{~dB}$ increments] to the contralateral ear at a rate of two stimuli per second.

Early exposure of rat pups. Litters of 3-4 P9 to P23 rat pups and their mothers were placed in a sound-shielded test chamber for 3-7 d. An $8 \mathrm{~h}$ light/16 h dark cycle was established. Two hundred and fiftymilliseconds-long $7 \mathrm{kHz}$ pure tones were presented to rat litters $24 \mathrm{~h} / \mathrm{d}$ at a sound level of $70 \mathrm{~dB}$ SPL with $500 \mathrm{~ms}$ quiet intervals. A $40 \mathrm{~Hz}$ amplitude modulation, at a depth of $1(100 \%)$ was added to the tone to augment its richness and salience. No distortion or significant harmonic signal was found in the chamber when a tonal stimulus was delivered. In addition, there was no abnormality in the behavior of either the mother or pups during monotone exposure. The weights of all pups and mothers were monitored continuously. There was no weight loss compared with naive rats, indicating normal lactation. The activities during wakefulness and the sleep behavior of the rat were normal (i.e., revealed no signs of stress).

Data analysis. The characteristic frequency (CF) of a cortical site was defined as the frequency at the tip of the tuning curve. When a tuning curve had a broad tip or multiple peaks, the median frequency at the threshold intensity was chosen as the CF. Response bandwidths $20 \mathrm{~dB}$ above threshold (BW20) were defined for all sites. For multipeaked tun- ing curves, the response bandwidth was defined as the range from the lowest to the highest frequency at $20 \mathrm{~dB}$ about the most sensitive tips that activated the cortical site, possibly encompassing the frequencies in a trough of the tuning curve that did not activate the cortical neurons. The response latency was defined as the time from stimulus onset to the earliest response observed in the PSTH containing pooled responses for all frequency-intensity combinations. The CF, threshold and BW20 were determined by direct visualization of the tuning curve in the MatLab environment (The MathWorks, Natick, MA) using custom routines.

To generate cortical maps, Voronoi tessellation ("voronoi" is a Matlab function) was performed to create tessellated polygons, with the electrode penetration sites at their centers. Each polygon was assigned the characteristics (e.g., CF) of the corresponding penetration site. In that way, every point on the surface of the auditory cortex could be linked to the characteristics experimentally derived from a sampled cortical site that was closest to that point. The boundaries of the primary auditory cortex were functionally determined using the following criteria: (1) primary auditory neurons generally have a continuous, single-peaked, $\mathrm{V}$-shaped receptive field, and (2) CFs of the Al neurons are tonotopically organized with high frequencies represented rostrally and low frequencies represented caudally (Bao et al., 2003b).

The tonotopic axis of the previously described CF maps was calculated by rotating the map to make horizontal a linear function fit of the penetration coordinates, using a least-squares method. After rotation, new $x$ coordinates of penetrations in each rat were normalized to be within a range from 0.0 to 1.0 , and penetration sites were plotted according to their CFs and $x$ coordinates.

The rate (spike count)/level function at each recorded site was calculated by averaging responses to three frequencies centered on the $\mathrm{CF}$ (spanning 0.3 octave) for stimuli between 0 and $70 \mathrm{~dB}$ SPL. The spike rate was then normalized to the rate at the first inflection point (point at which the first derivative of the function is at an extremum) of the rate/ level function (see Fig. $8 B, C$ ). The segment of the rate/level function between the inflection point and the end of the data ("last segment") was then fit using a least-squares method. A response was designated as nonmonotonic if that last segment had a slope $<-0.5 \% / \mathrm{dB}$ and spanned at least 10 dB (Ramachandran et al., 1999; Bonham et al., 2004).

Unless specified otherwise, statistical significance was assessed using unpaired two-tailed $t$ tests. Data are presented as mean \pm SE.

\section{Results}

\section{Opening of the $\mathrm{CP}$}

To determine the precise timing of the opening of the $\mathrm{CP}$, rat pups were exposed to 3 consecutive days of $7 \mathrm{kHz}$ pure tones of 70 dB SPL intensity (see Materials and Methods) starting at P8, P9, and P11 (Fig. 1A). Tone-evoked responses within the auditory cortex were reconstructed to document stimulus-specific changes $7 \mathrm{~d}$ after the end of the exposure period, at P17, P18, and P20, respectively. Stimulus-induced plasticity was measured by obtaining complete CF maps of A1 (see Materials and Methods) and then calculating the percentage of the area of A1 tuned to 7 $\mathrm{kHz} \pm 0.3$ octave (Fig. $1 \mathrm{~A}$ ). The earliest exposure start date was $\mathrm{P} 8$, a date in postnatal development at which the auditory pathways are not activated by low-threshold air-conducted stimuli (Geal-Dor et al., 1993).

The extent of A1 cortical area tuned to $7 \mathrm{kHz} \pm 0.3$ octaves in exposed litters was compared with age-matched naive controls. No significant expansion was found in the group exposed from P8 to P10 (P8-P10 exposed, $17.5 \% \pm 1.3 \%, n=5$; total number of sites, 226; naive P17, $14.4 \pm 1.58 \%, n=4$; total number of sites, $176 ; p=0.21$ ). If the exposure started only $1 \mathrm{~d}$ later at P9 (P9 to $\mathrm{P} 11$ exposure) a significant expansion of $7-\mathrm{kHz}$-tuned sites was found (P9-P11 exposed, $25.7 \pm 1.4 \%, n=5$; total number of sites, 224; naive P18, $13.3 \pm 1.1 \%, n=4$; total number of sites, $205 ; p<0.001$ ). If the exposure spanned P11 to P13, the proportion of $\mathrm{Al}$ tuned to $7 \mathrm{kHz}$ was more than twice that of controls 
A

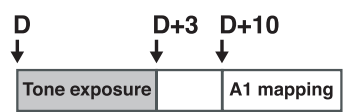

Opening of the critical period

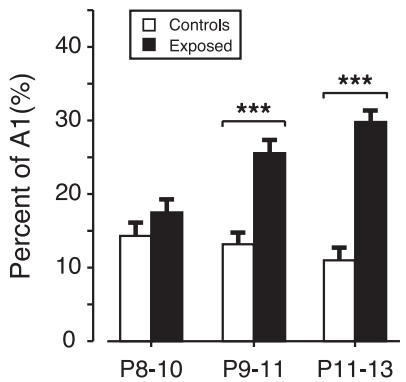

Period of exposure
B

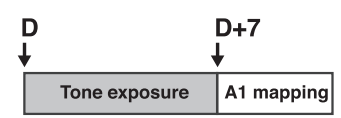

Closing of the critical period
A

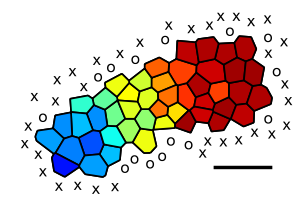

C

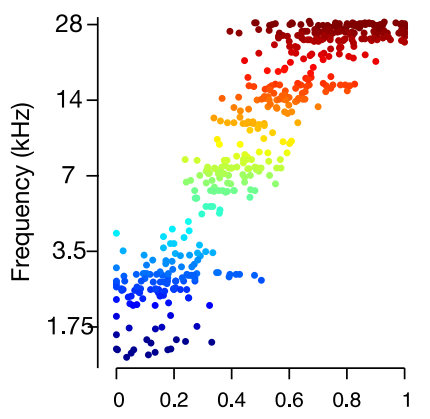

Normalized tonotopic axis
B

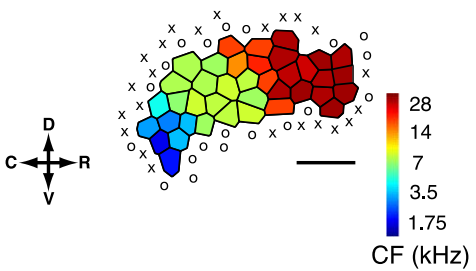

$7 \mathrm{kHz}$ exposed

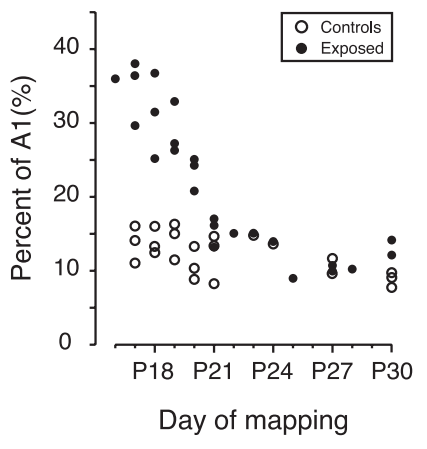

Figure 1. Opening and closing of the CP. A, Top, Experimental protocol used to determine the time of opening of the $C P$. Rats were exposed to $3 \mathrm{~d}$ of $7 \mathrm{kHz}$ pure tones starting at different postnatal days (D) and mapped $7 \mathrm{~d}$ after the end of the exposure period. Bottom, Percentage of A1 area with receptive fields tuned to $7 \mathrm{kHz} \pm 0.3$ octave as a function of the exposure period (P8-P10 period, $n=5 ; \mathrm{P} 9$-P11 period, $n=5 ; \mathrm{P} 11-\mathrm{P} 13$ period, $n=5$; naive, $n=4$ ). Values shown are mean $\pm S E .{ }^{* * *} p<0.001, t$ test. $\boldsymbol{B}$, Top, Experimental protocol used to determine the time of closing of the $\mathrm{CP}$. Rats were exposed to $7 \mathrm{~d}$ of $7 \mathrm{kHz}$ pure tones starting at different postnatal days $(D)$ and mapped $7 \mathrm{~d}$ later $(D+7)$. Bottom, Percentage of $A 1$ area with receptive fields tuned to $7 \mathrm{kHz} \pm 0.3$ octave, with respect to the day of mapping $(D+7)$ (exposed, filled circles, $n=25$; naive, open circles, $n=21$ ).

(P11-P13 exposed, $30.0 \pm 1.3 \%, n=6$; total number of sites, 335; naive $\mathrm{P} 20,11.1 \pm 1.2 \%, n=5$; total number of sites, $219 ; p<$ $0.001)$. This effect was not significantly different from the maximal effect obtained with the $7 \mathrm{~d}$ exposure (see below).

\section{Closing of the CP}

To estimate the date at which experience-dependent plasticity ends and to evaluate the impact of a longer exposure period, we exposed rat pups to $7 \mathrm{kHz}$ tones for seven consecutive days starting at P9 or later (Fig. 1B). This exposure was staggered over different postnatal periods and the effect of this exposure on 7 $\mathrm{kHz}$ representation in $\mathrm{A} 1$ was measured at the end of the seventh day of exposure. The maximal effect was obtained in litters mapped at P16, P17, P18, and P19 (exposure start date of P9, P10, $\mathrm{P} 11$, and P12, respectively). Rats in these litters had a highly significant, greater than twofold expansion of A1 sites tuned to the stimulus frequency \pm 0.3 octaves (exposed, mapped at P16P19 average, $32.1 \pm 1.50 \%, n=10$; total number of sites, 406 ; age-matched naive average, $13.9 \pm 0.68 \%, n=9$; total number of sites, $426 ; p<0.0001)$. A representative CF map of one naive rat and a typical sound-exposed rat are shown in Figure $2 A, B$. For litters mapped at P20, a significant over-representation of sites tuned to $7 \mathrm{kHz}$ was still found (exposed, mapped at P20, $23.4 \pm$ $1.32 \%, n=3$; total number of sites, 121 ; naive, mapped at P20, $10.8 \pm 1.31 \%, n=3$; total number of sites, $131 ; p<0.01)$. No significant distortion of frequency representation in A1 was observed if the exposure was started at P14 or later (controls, $n=7$; exposed, $n=11 ; p>0.2$ ).

\section{$\mathrm{CP}$ exposure drives persistent changes in A1 tuning}

The cortical alterations caused by sensory deprivation or disturbance during the $\mathrm{CP}$ are generally considered to be long lasting
Figure 2. Effect of $(P$ exposure on $\mathrm{A} 1$ characteristic-frequency maps. $A, B$, Representative $A 1$ CF map from a naive P18 rat $(A)$ and from a rat exposed to $7 \mathrm{kHz}$ pure tones throughout the critical period, then mapped at $P 18(\boldsymbol{B}) . \boldsymbol{C}, \boldsymbol{D}$, Distribution of tuning curve $C$ Fs plotted against a normalized tonotopic axis (see Materials and Methods) in litters of naive controls $(\boldsymbol{C})$ and litters exposed during the $C P(D)$. Note the increased number of recording sites at which neurons were tuned to $7 \mathrm{kHz}$ in the exposed group (arrow). Scale bar, $0.5 \mathrm{~mm}$. X, Unresponsive cortical site; 0 , non-Al cortical site (see Materials and Methods); D, dorsal; C, caudal; R, rostral; V, ventral. (exposed, $n=9$; recorded sites, 458; naive, $n=9$; recorded sites, 479 .)

(Hensch, 2004). To evaluate the long-term impact of monotone exposure during the $\mathrm{CP}$, we exposed rats to $5 \mathrm{~d}$ of pulsed $7 \mathrm{kHz}$ pure tones starting $1 \mathrm{~d}$ before the onset of the CP (P10) and ending $1 \mathrm{~d}$ after its closure (P14) (Fig. 3). The rats were then returned to a neutral auditory environment and mapped at P60. The percentage of the area of $\mathrm{A} 1$ tuned to $7 \mathrm{kHz} \pm 0.3$ octave was measured and compared with aged-matched controls. We found that $>6$ weeks after the end of the exposure, at P60, rats in the exposed group had a significantly enlarged $7-\mathrm{kHz}$-tuned area compared with controls (exposed average, $27 \pm 4.6 \%, n=4$; total number of sites, 195; controls average, $14.9 \pm 1.9 \%, p<$ $0.01, n=4$; total number of sites, 211). The magnitude of the effect observed here was not significantly different from the distortion found with the 7-d-exposure litters. Of note, the area of A1 tuned to $3 \mathrm{kHz}$ was significantly lower in exposed litters compared with controls (exposed average, $6.2 \pm 0.7 \%, n=4$; controls average, $11.1 \pm 0.8 \% ; p<0.01)$.

\section{$\mathrm{CP}$ exposure to $7 \mathrm{kHz}$ causes an under-representation of low frequencies in $\mathrm{Al}$}

How does $7 \mathrm{kHz}$ tone exposure during the $\mathrm{CP}$ impact the total area of the functionally defined A1, and how does it impact the representations of other frequencies within A1? Results were obtained from litters exposed for $7 \mathrm{~d}$ to $7 \mathrm{kHz}$ and were grouped by day of mapping in $3 \mathrm{~d}$ bins centered at $\sim$ P17, P20, P24, and P30. Our exposure protocol led to a significant $19 \%$ reduction in total A1 size for animals mapped at P17 $\pm 1 \mathrm{~d}$ (exposed average, $1.037 \pm 0.04 \mathrm{~mm}^{2}, n=11$; naive average, $1.277 \pm 0.04 \mathrm{~mm}^{2}, n=$ 9 ; $p<0.01)$. A significant lesser reduction in A1 size was still found for litters mapped at P20 $\pm 1(p<0.05)$ but not for rats mapped after P20. 
A

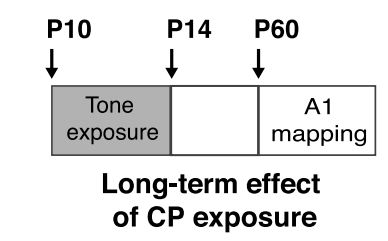

CF representation difference

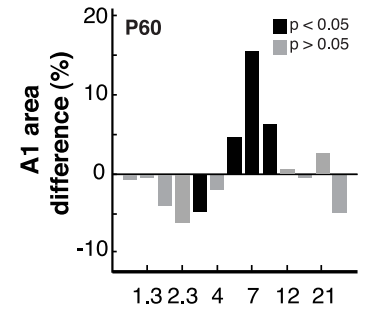

Characteristic frequency $(\mathbf{k H z})$
B
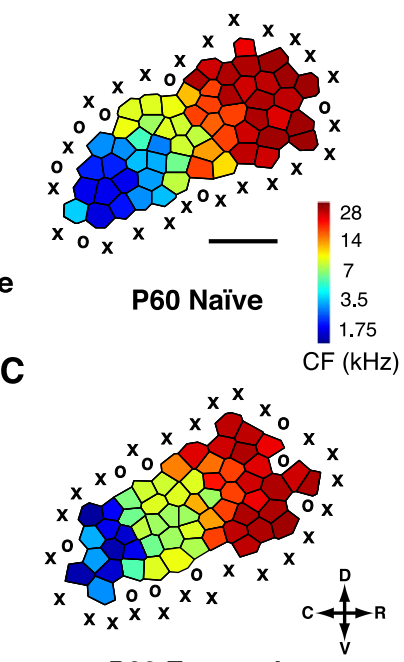

P60 Exposed
Figure 3. Long-term effect of $C P$ exposure on $\mathrm{A} 1$ tuning. $A$, Top, Experimental protocol used to evaluate the long-term impact of $\mathrm{CP}$ exposure on $\mathrm{A} 1$ tuning. Rats were exposed to $5 \mathrm{~d}$ of $7 \mathrm{kHz}$ pure tones starting $1 \mathrm{~d}$ before the opening of $C P(P 10)$ and ending $1 \mathrm{~d}$ after the closure (P14) to finally be mapped at $P 60$. Bottom, Difference in frequency tuning between exposed and naive rats expressed as $\mathrm{A} 1$ percentage and separated by $\mathrm{C} F$ for litters mapped at $\mathrm{P} 60$ (controls, $n=4$; exposed, $n=4)$. $\boldsymbol{B}, \boldsymbol{C}$, Representative $\mathrm{A} 1$ (F maps from a naive $\mathrm{P} 60$ rat $(\boldsymbol{B})$ and from a rat exposed to $7 \mathrm{kHz}$ pure tones throughout the critical period, between P10 and P14, and then mapped at $P 60$ (C). Values shown are mean $\pm S E .{ }^{* *} p<0.01, t$ test. D, Dorsal; $C$, caudal; $R$, rostral; $V$, ventral.

An expansion of 7-kHz-tuned sites in the context of a reduction in the size of $\mathrm{Al}$ in exposed animals has to occur at the expense of sites tuned to other frequencies. That fact can be visualized in Figure 2, $C$ and $D$, where the $C F$ of each tuning curve recorded within $\mathrm{Al}$ is plotted against a normalized tonotopic axis (see Materials and Methods). Figure $2 D$ shows pooled recordings from exposed litters mapped at P17, P18, and P19 $(n=9$; total sites, $n=458$ ) and Figure $2 C$ shows the distribution of tuning curve CFs from pooled age-matched controls ( $n=9$; total sites, $n=479$ ). Examination of the CF distribution in the exposed group immediately reveals an over-representation of sites tuned to $7 \mathrm{kHz}$ and a relative under-representation of sites tuned to immediately adjacent frequencies, especially in the $2-5 \mathrm{kHz}$ range. These differences were quantified and the results can be visualized in Figure $4 A-C$. For this analysis, litters exposed to $7 \mathrm{~d}$ of $7 \mathrm{kHz}$ tones were grouped in $3 \mathrm{~d}$ bins centered $\sim \mathrm{P} 17, \mathrm{P} 20$, and P23. For rats mapped at P17 (exposed, $n=13$; number of sites, 527; naive, $n=11$; number of sites, 512 ), there was a significant near $50 \%$ reduction of the area of A1 tuned to $2.3 \mathrm{kHz}$ (exposed average, $9.8 \pm 1.7 \%, n=13$; naive, $17.1 \pm 1.6 \%$; $p<0.01$ ) and $3.0 \mathrm{kHz}$ (exposed average, $10.6 \pm 1.7 \%, n=13$; naive, $15.7 \pm$ $1.7 \%, n=11 ; p<0.05)$. A1 tuning to higher frequencies was also found to be reduced between 12 and $21 \mathrm{kHz}$ but the difference was not statistically significant (for $21 \mathrm{kHz}$ tuned sites, exposed average, $16.0 \pm 2.5 \%, n=13$; naive, $22.2 \pm 2.1 \%, n=11 ; p=$ 0.08 ). For rats mapped at P20 (exposed, $n=6$, number of sites, 251; naive, $n=5$; number of sites, 219), a lesser but significant reduction in A1 tuning to 2.3 and $4.0 \mathrm{kHz}$ was still found (for 2.3 $\mathrm{kHz}$ tuned sites, exposed average, $12.8 \pm 1.4 \%, n=6$; naive, $20.0 \pm 2.6 \%, n=5 ; p<0.05)$. No significant difference was found in $\mathrm{A} 1$ frequency representation for litters mapped at $\mathrm{P} 23$ or later $(n=11$ for exposed, number of sites, $461 ; n=7$ for controls, number of sites, 291).
A

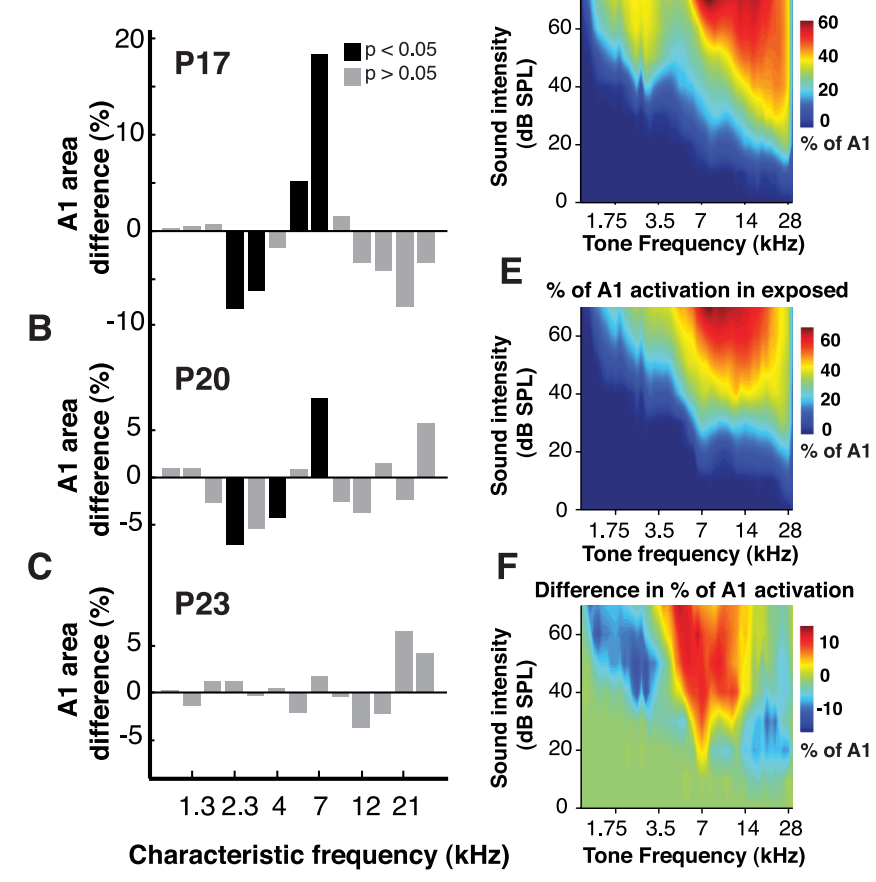

Figure 4. Effect of $C P$ exposure on frequency representation. $A-C$, Difference in frequency tuning between exposed and naive rats expressed as $A 1$ percentage and separated by $C F$ for litters mapped at P17 (A), P20 (B), and P23 (C). These rats were exposed for $7 \mathrm{~d}$ of $7 \mathrm{kHz}$ pure tones and mapped on the seventh day of exposure (exposed, $n=9$; naive, $n=9$ ). $\boldsymbol{D}, \boldsymbol{E}$, Percentage of $A 1$ activated by every frequency-intensity combination used for mapping in naive (D) and $(P$ tone-exposed $(\boldsymbol{E})$ rats. $\boldsymbol{F}$, Difference in percent of activation between $(P$ toneexposed and naive rats (exposed, $n=9$; naive, $n=9$ ). ${ }^{* *} p<0.01$, $t$ test.

Changes in CF maps as shown above addressed the effect of the $7 \mathrm{kHz}$ exposure on RF tuning at threshold, the CF, but did not look at response changes at different sound intensity levels. For this analysis, the proportion of the area of Al showing toneevoked spiking was calculated for every sound-intensity combination used for mapping (Fig. $4 D-F$ ). Litters that received a $7 \mathrm{~d}$ exposure ending at P18, P19, and P20 were grouped and compared with age-matched naive controls (number of exposed rats, 9; total sites, $n=458$; number of exposed rats, 9; total sites, $n=$ 479). As expected, a significant increase in activity was found in the exposed group for frequencies close to the exposure stimulus, between 4.3 and $8.0 \mathrm{kHz}$, for intensities between 30 and $70 \mathrm{~dB}$ SPL. For example, at $60 \mathrm{~dB}$ SPL, on average $57.2 \pm 2.9 \%$ of A1 was activated by a $7.0 \mathrm{kHz}$ tone in the exposed litters whereas $43.7 \pm$ $3.4 \%$ of $\mathrm{A} 1$ was activated by the same tone in controls $(p<0.05)$ (Fig. $4 D-F$ ). In contrast, cortical activity in $\mathrm{A} 1$ was found to be significantly decreased for frequencies ranging from 1.4 to 4.9 $\mathrm{kHz}$ between 30 and $60 \mathrm{~dB}$ SPL. For instance, at $50 \mathrm{~dB}$ SPL, on average $18.7 \pm 11.1 \%$ of Al was activated by a $2.47 \mathrm{kHz}$ tone in the exposed litters whereas $32.3 \pm 7.1 \%$ of $\mathrm{A} 1$ was activated by the same tone in controls $(p<0.05 ; n=9)$. A significant $9.6 \%$ decrease in cortical activity was also found for higher frequencies $(18.5-21.3 \mathrm{kHz})$ but only at $30 \mathrm{~dB}$ SPL in the exposed group compared with controls.

The $\mathrm{CP}$ for spectral selectivity in $\mathrm{Al}$ coincides with the epoch of maturation of simple (tone-evoked) excitatory auditory responses

In other rat sensory cortices like the barrel cortex (Rice and Van der Loos, 1977; Schlaggar and O'Leary, 1993; Stern et al., 2001) 
and the primary visual cortex (Berardi et al., 2000), the CP for experiencedependent plasticity is temporally linked to the maturation of peripheral sensory end organs and the emergence of cortical responses to sensory input. To assess the state of maturity of the primary auditory cortex of the rat during the $\mathrm{CP}$, we examined the response characteristics of the primary auditory cortical neurons in naive rat pups between $\mathrm{P} 10$ and $\mathrm{P} 60(n=3$ between $\mathrm{P} 10$ and $\mathrm{P} 14 ; n=6$ for P17; $n=4$ for P30; $n=4$ for P60). The sound intensity thresholds, onset latencies and bandwidth at $20 \mathrm{~dB}$ above threshold (BW20s) of pure tone-evoked cortical responses were measured in developing rats and compared with P30 and P60 values (Figs. $5 A-K, 6 A-D)$. For this analysis, cortical sites were divided in low CF sites (1-5.6 $\mathrm{kHz})$ and high CF sites $(5.7-30 \mathrm{kHz})$.

No tone-evoked responses could be obtained in 10-d-old rats for pure tones or noise bursts of $80 \mathrm{~dB}$ SPL or less. At P11, the earliest age at which tone-evoked responses could be obtained in the cortex, responses were found only for relatively high-intensity stimuli (threshold average, $57.8 \pm 4.3 \mathrm{~dB}$ SPL; number of sites, 44) and the average cortical area responding to tonal stimuli was $<25 \%$ of the average P60 A1 area (P11 area average, $0.30 \pm$ $0.087 \mathrm{~mm}^{2}$; P60 average, $1.24 \pm 0.1 \mathrm{~mm}^{2}$; $p<0.001$, ANOVA). Receptive field CFs were limited to frequencies between 6.0 and $9.9 \mathrm{KHz}$. Rapid changes occurred over the subsequent $3 \mathrm{~d}$, and by P14 the auditory cortex had adult-like excitatory responses to simple tones and A1 could be readily identified using conventional criteria (see Materials and Methods). At P14, A1 map size (Fig. 6D) did not differ from A1 size recorded at P60 (P14 average, $1.29 \pm 0.069 \mathrm{~mm}^{2}$; P60 average, $1.24 \pm$ $0.1 \mathrm{~mm}^{2} ; p>0.2$, ANOVA). Thresholds (Fig. $6 B$ ) of high CF sites at P14 were not different from the P60 value (P14 high CF sites average, $19.0 \pm 4.3 \mathrm{~dB}$ SPL; number of sites, 87; P60 high CF sites average, $14.6 \pm 1.0 \mathrm{~dB}$ SPL; number of sites, 146 ; $p>0.2$, ANOVA). At P17, thresholds of low CF sites were similar to P60 values (P17 low CF sites average, $40.1 \pm 6.8 \mathrm{~dB}$ SPL; number of sites, 41; P60 low CF sites average, $40.5 \pm 1.2 \mathrm{~dB}$ SPL; number of sites, $65 ; p>0.2$, ANOVA). Low CF sites (as defined above) could be recorded starting only at P13, and the range of tuning curve CFs at P14 was equivalent to the range found in P30 animals $(1.5-30 \mathrm{kHz}$; note that only frequencies between 1 and $30 \mathrm{kHz}$ were tested).

Response BW20s were measured be-
A

B

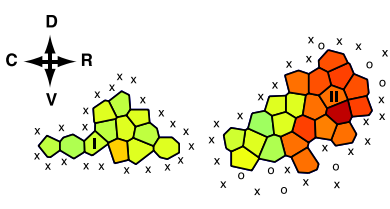

P11

P12

C

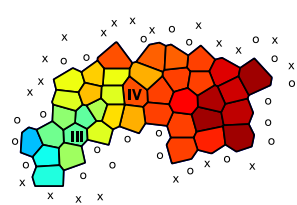

P13
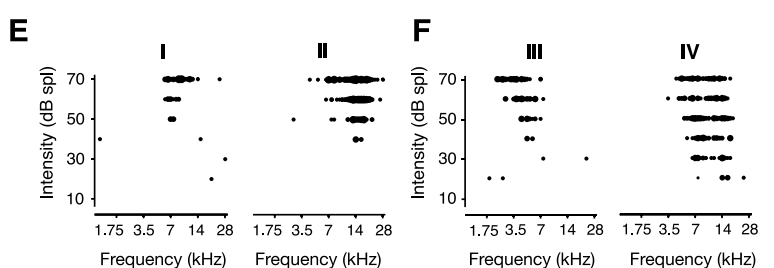

D

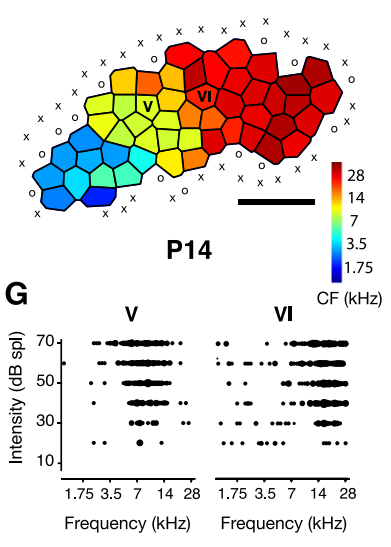

H
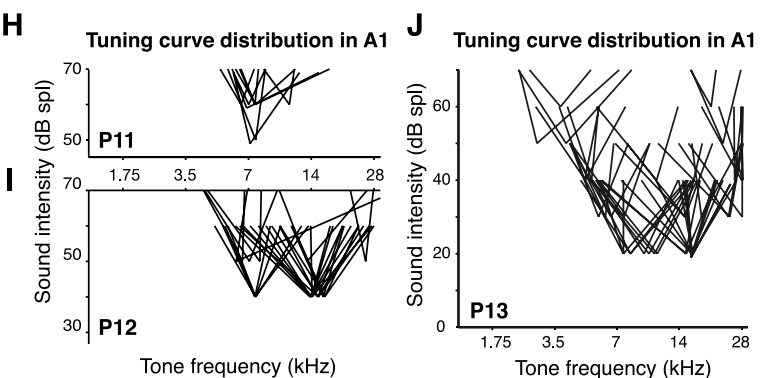

K Tuning curve distribution in A1

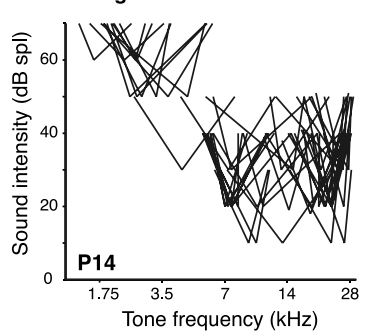

Figure 5. Maturation of excitatory tone-evoked responses in $A 1$ during the CP. A-D, Representative A1 CF maps obtained at different postnatal ages in naive controls between P11 and P14. Scale bar, $0.5 \mathrm{~mm}$. X, Unresponsive cortical site; 0 , non-Al cortical site; D, dorsal; C, caudal; $R$, rostral; $V$, ventral. $\boldsymbol{E}-\mathbf{G}, I-V I$, Sites where the receptive fields were recorded. $\boldsymbol{H}-\boldsymbol{K}$, Repertoire of tuning curves in P11 $(\boldsymbol{H}), \mathrm{P} 12(\boldsymbol{I}), \mathrm{P} 13(\boldsymbol{J})$, and P14 $(\boldsymbol{K})$ naive rats. The apex of each pair of joined lines indicates the threshold and $\mathrm{CF}$ recorded at each particular penetration site. The separation $20 \mathrm{~dB}$ above that apex is the BW20 described in Materials and Methods and Results. ( $n=3$ for each postnatal day between P10 and P14.)

A

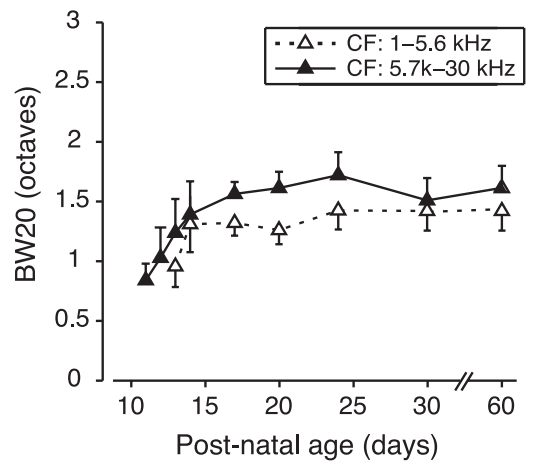

C

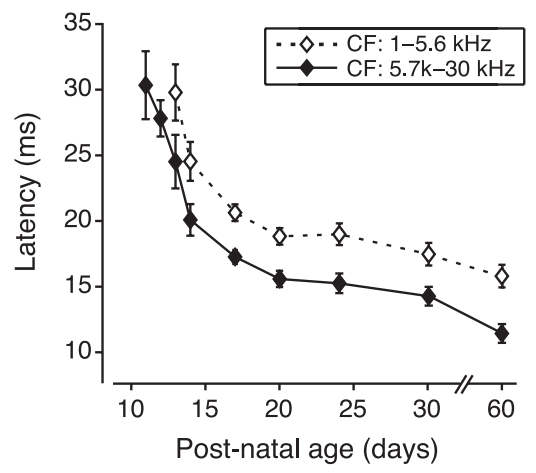

B

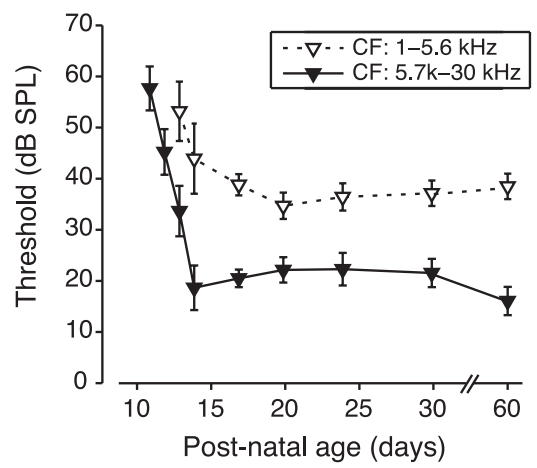

D

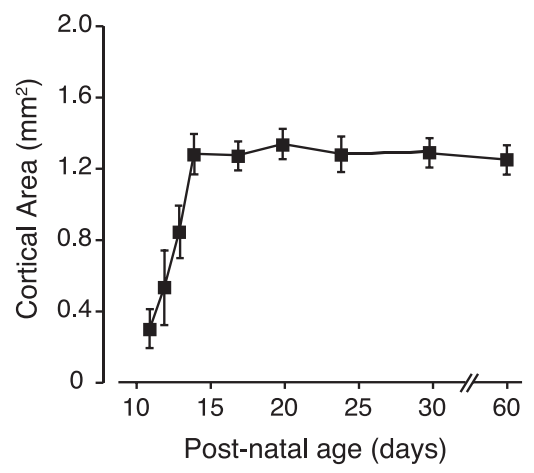

Figure 6. Evolution of response characteristics in $\mathrm{A} 1$ as a function of age. $\boldsymbol{A}$, Bandwidths at $20 \mathrm{~dB}$ above threshold. $\boldsymbol{B}$, Thresholds. C, Latencies. D, Total A1 cortical area. Open symbols, Cortical sites with a CF between $1-5.6 \mathrm{kHz}$; closed symbols, cortical sites with a CF between $5.7-30 \mathrm{kHz}$; age, day of mapping; all data are from naive (nontone-exposed) rats ( $n=21$ ). 
tween P11 and P60 (Fig. 6A). BW20s were significantly smaller in P11 litters for high CF cortical sites, and reached stable (P60) values at $\sim \mathrm{P} 17$ for low and high $\mathrm{CF}$ sites (P11 high CF sites average, $0.84 \pm 0.14$ octave; number of sites, 33 ; $\mathrm{P} 17$ high $\mathrm{CF}$ sites average, $1.57 \pm 0.10$ octave; number of sites, $91 ; p<0.01$ ANOVA). A similar tendency was found for low CF sites (P13 low CF sites average, $0.96 \pm 0.19$ octave; number of sites, 29; P17 low CF sites average, $1.33 \pm 0.11$ octave; number of sites, $41 ; p<0.05$ ANOVA). Onset latencies (Fig. 6C) for high and low CF sites were markedly prolonged in $\mathrm{P} 11-\mathrm{P} 13$ rats and declined rapidly up to P20 and then slowly decreased up to P60 (P11 high CF sites average, $30.3 \pm 2.6 \mathrm{~ms}$; number of sites, 33; P30 high CF average, $12.6 \pm 0.5 \mathrm{~ms}$; number of sites, $150 ; p<0.01$, ANOVA; P13 low CF average, $29.8 \pm 2.1 \mathrm{~ms}$; number of sites, 29; P30 low CF average, $15.8 \pm 0.6 \mathrm{~ms}$; number of sites, $45 ; p<0.01$, ANOVA).

Exposure to $7 \mathrm{kHz}$ pure tones during the $\mathrm{CP}$ (P11-P13) or up to P30 had no significant effect on tuning bandwidth, thresholds or latencies for sites tuned to a wide range of frequencies. (Fig. $7 A-C)$.

\section{Pure tone exposure increased the number of sites with nonmonotonic rate/sound level functions}

Rate (spike count)/sound level (intensity) functions were determined for every recorded site in rats exposed between P11 and P13 (total number sites, 406) and in age-matched controls (total number sites, 438). A recorded site was defined as being nonmonotonic if the slope of the last segment (see Materials and Methods) was $-0.5 \% / \mathrm{dB}$ or less (Bonham et al., 2004). Results are shown in Figure 8. The proportion of nonmonotonic sites was greater in rats exposed to $7 \mathrm{kHz}$ during the $\mathrm{CP}$ compared with controls $(15.8 \pm 1.2$ vs $7.6 \pm 1.9 \% ; p<0.01 ; n=10$ for exposed; $n=9$ for controls). If recorded sites were separated in $7 \mathrm{kHz} \pm$ 0.3 octave or non-7-kHz-tuned sites, this effect was still present and significant for both groups (7-kHz-tuned sites, $18.0 \pm 2.7 \%$ vs $6.4 \pm 2.4 \%$ for controls, $p<0.01$; non- $7 \mathrm{kHz}$ sites $14.9 \pm 1.4 \%$ vs $7.8 \pm 2.0 \%$ for controls, $p<0.01$; exposed group: total of 7 $\mathrm{kHz}$ sites, 136; total of non-7 kHz sites, 270; control group: total of 7-kHz-tuned sites, 70; total of non-7 kHz sites, 368). A small but significant difference was also found between the mean slope per animal of the last segment of the rate/level function in litters exposed during the CP and controls ( 0.36 vs $0.69 \% / \mathrm{db} ; p<0.05$; $n=10$ for exposed; $n=9$ for controls). That difference was accounted for by $7 \mathrm{kHz}$ sites $(0.20 \% / \mathrm{dB}$ for exposed vs $0.47 \% / \mathrm{db}$ for naive; $p<0.05$; exposed group: total of $7 \mathrm{kHz}$ sites, 136 ; total of non-7 kHz sites, 270; control group: total of 7-kHz-tuned sites, 70; total of non-7 kHz sites, 368). No difference was recorded for non-7 kHz locations.

\section{Discussion}

Definition of the critical period window for the rat primary auditory cortex, A1

Continuous exposure to pulsed $7 \mathrm{kHz}$ tonal stimuli in rat pups reared in a quiet environment resulted in a large expansion of sites tuned to that frequency in the primary auditory cortex (A1), only if that exposure occurred between P11 and P13. As for V1 CP plasticity, the effects observed here were persistent, still demonstrable several weeks after the end of the sensory manipulation. This is the first study determining the onset, duration, and offset of the critical period (CP) in $\mathrm{A} 1$, in any mammalian species.

The magnitudes of changes observed were similar to those seen in previous studies in which the sound exposure extended from before to several weeks after closure of this narrow critical period window (Zhang et al., 2001). Early postnatal sound
A

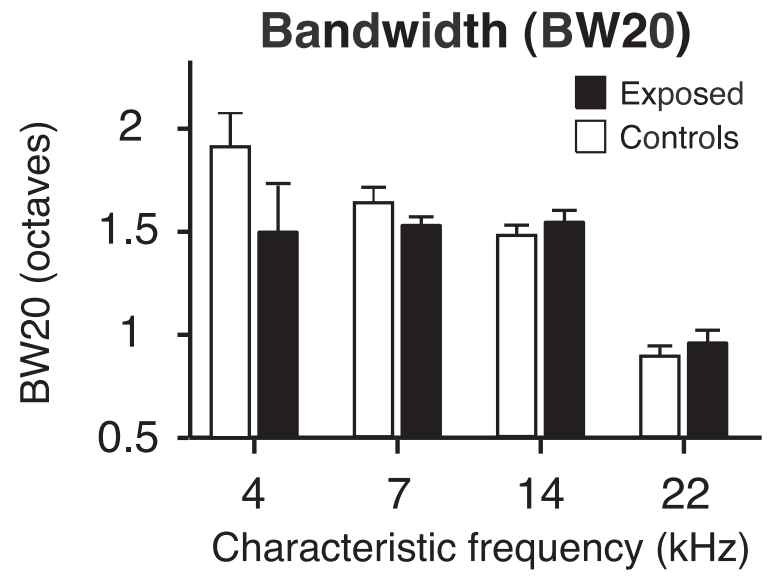

B

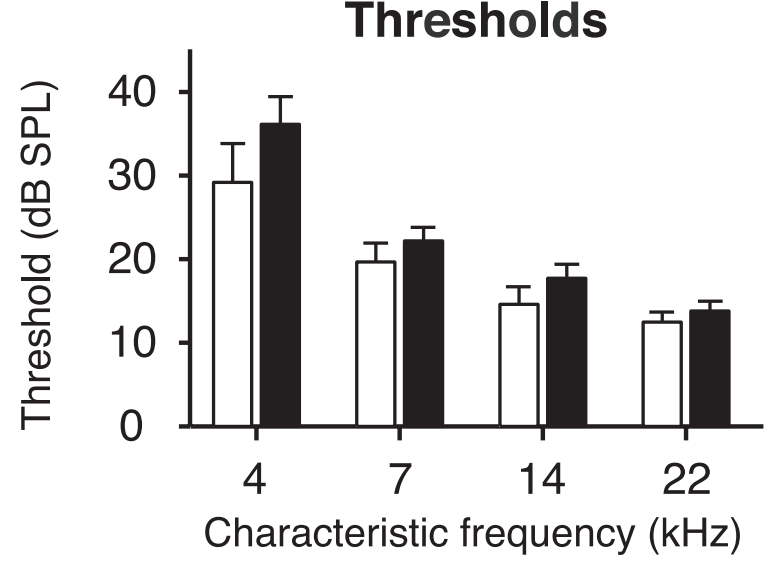

C

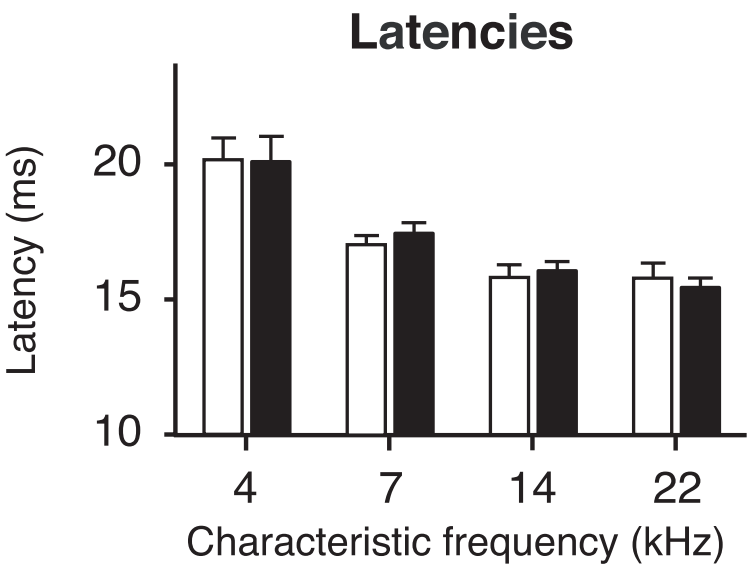

Figure 7. Effect of $(P$ exposure on response characteristics in $A 1$. $A-C$, Bandwidths at $20 \mathrm{~dB}$ above threshold $(\boldsymbol{A})$, thresholds $(\boldsymbol{B})$, and onset latencies $(\boldsymbol{C})$ separated by characteristic frequency in rats exposed (dark bars) throughout the $\mathrm{CP}(n=9)$ and in naive (light bars) rats $(n=9)$.

exposure-driven plasticity has also been documented in the auditory brainstem (Clopton and Winfield, 1976; Sanes and Constantine-Paton, 1983; King et al., 1988; Poon and Chen, 1992; Knudsen, 1998), and it is possible that the distortions in spectral representation recorded here at the cortical level largely reflect subcortical changes. However, given the rapidity of these plastic changes, and given compelling evidence that the auditory cortex, via top-down control controls the spectral selectivity of neurons in the thalamus and inferior colliculus (Yan and Suga, 1996, 1998; Zhang and Suga, 1997; Rauschecker, 1998), addi- 
tional studies shall have to be conducted to disambiguate the cortical versus subcortical origins of this plastic remodeling of $\mathrm{A} 1$ responses. Until such studies are completed, the most conservative approach is to view cortically recorded changes as a probable product of early auditory system plasticity, contributed to by coordinated cortical and brainstem changes.

The relatively short A1 CP we evidenced is comparable in length to the $\mathrm{CP}$ in the rat somatosensory (S1 barrel-field) cortex (Rice and Van der Loos, 1977; Schlaggar and O'Leary, 1993). However, it is much shorter than the $\sim 4$-week-long $\mathrm{CP}$ recorded in the rat's primary visual cortex, V1 (Fagiolini et al., 1994). In V1, BDNF (Hanover et al., 1999) and the development of GABAergic projections have been shown to play a key role in the onset of the critical period (Fagiolini and Hensch, 2000; Fagiolini et al., 2004). Similarly, dark-rearing delays the onset of the critical period (Mower, 1991; Iwai et al., 2003). Strikingly, once triggered, the critical period is always of a fixed duration. Factors that may close the critical period have recently involved the extracellular matrix, such as perineuronal nets (Pizzorusso et al., 2002, 2006) and myelin-related signals (McGee et al., 2005).

As in $\mathrm{V} 1$, the development of normal tonotopic organization and frequency tuning in A1 appears to require exposure to normally patterned sound inputs (Zhang et al., 2002) (for an example of inferior colliculus, see Sanes and Constantine-Paton, 1985). Even more compellingly, continuous white-noise rearing, like dark-rearing for $\mathrm{V} 1$, results in an indefinite prolongation of the A1 CP window (Chang and Merzenich, 2003, 2005) suggesting that the structure of the auditory environment plays an important role in the gating of the critical period. The duration and offset of the critical period in A1 might therefore be substantially influenced by the complexity and variability of sounds present in the early postnatal environment and an exposure using more complex "natural" stimuli could lead to a different, potentially longer CP window estimation. In contrast, a monotonous, highly structured stimulus as the one used in this study could have promoted an accelerated closure of the $\mathrm{CP}$ by massively engaging auditory cortical neurons. It is worth mentioning that Zhang et al. (2002) examined the effect of an early broadband stimulus exposure and found that a $20 \mathrm{~d}$ pulsed-noise exposure significantly degraded frequency tuning in A1 only if the exposure occurred between P8 and P28 but not after P30.

Despite the fact that mammals possess a precise frequencybased cortical representation of sound, pure tones are not common in natural auditory environments. Although facilitating greatly the precise identification of stimulus-specific changes in the developing cortex, the use of pure tones in our exposure protocol could have prevented us from evidencing a longer plasticity window for higher-order neuronal properties. It should be noted that two reported series of experiments have described the consequences of rearing rats in a very complex stimulus environment extending from before hearing onset (before P10) to far beyond the end of the critical period as determined in this study.
B
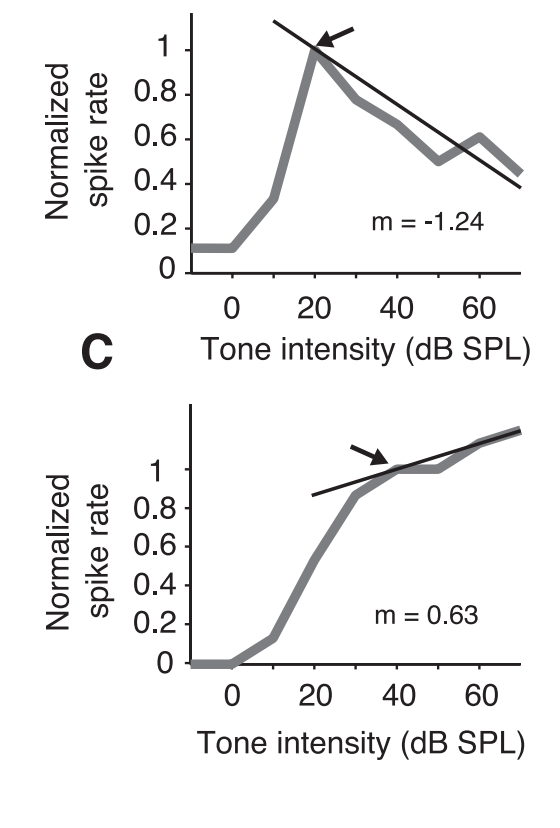

B

Tone intensity (dB SPL)

\section{$\begin{array}{ccc}\text { All } & 7 k & \text { Non-7k } \\ \text { Sites } & \text { Sites } & \text { Sites }\end{array}$}

Figure 8. (P exposure increases nonmonotonicity. A, Proportion of recorded sites per animal with nonmonotonic rate-level functions (see Materials and Methods) in $A 1$ in exposed (E) and naive controls (C) grouped by frequency tuning (left, all sites tative rate-level functions of a nonmonotonic site $(\boldsymbol{B})$ and monotonic site $(\boldsymbol{C})$ recorded in a tone-exposed animal and a naive segment between inflection point (arrow) and end of data. ${ }^{* *} p<0.01, t$ test.

Both revealed the emergence of a higher preponderance of inseparable spectrotemporal feature-selective neurons in A1, and both recorded what appeared to be an intermediate stage of plasticity at the end of complex sound exposure (P28 to P30) with still greater environmental-sound-stimulus-specific changes recorded in P80-P100 (infant-exposed) rats (Bao et al., 2003a; Nakahara et al., 2004b) (S. Bao and H. Nakahara, personal communication). Nakahara et al. (2004a,b) have also demonstrated that early exposure to sequenced sound stimuli results in the emergence of combination-selective (sequence-specific) A1 neuronal responses. Previous studies have shown that the maturation of temporal modulation response characteristics dependent on a progressive change in inhibitory time constants, and the maturation of side-band inhibition contributing to the sharpening of response tuning in $\mathrm{A} 1$ are delayed in their emergence to an epoch well beyond (up to P35-P45) the critical period time window we described here (Chang et al., 2005). These examples suggest, like in V1, the existence of different successive CP epochs for distinct aspects or complexity of sound representation in the auditory system and the brief CP window for spectral tuning we described could be an essential initial foundation for the subsequent consolidation of more complex sound representations. Maturity of A1 defined in the current study by simple measures of toneevoked tuning, growth of response magnitude versus intensity functions, and tonotopy may therefore only incompletely describe the evolution of the functional A1 by sound exposure in the $\mathrm{CP}$ epoch. Ongoing studies are now directed toward determining the influence of sound input quantities, complexities, and variability, as modifiers of A1 CP onset, duration, and closure.

Paradoxically, overall A1 area was reduced in sound-exposed litters, despite the elaboration of representation of the environmentally delivered tonal stimulus. Exposure to $7 \mathrm{kHz}$ tones during the $\mathrm{CP}$ also resulted in a significant under-representation of 
immediately lower sound frequencies. Similar competitive effects have been recorded in earlier CP sound exposure studies (Zhang et al., 2001; Chang and Merzenich, 2003, 2005; Nakahara et al., $2004 a, b)$ and are analogous to the cortical rearrangements seen after monocular deprivation in V1 (Shatz and Stryker, 1978; LeVay et al., 1980) or after digit amputation in S1 (Merzenich et al., 1984). In V1, the preferential stabilization of certain thalamocortical connections appears to be tightly linked to correlated electrical activity during the CP (Wiesel and Hubel, 1963; Guillery and Stelzner, 1970; Shatz, 1990). The highly structured stimulus we used could have favored the development of cortical areas receiving $7 \mathrm{kHz}$ input over those mainly activated by non- $7 \mathrm{kHz}$ stimuli. Similarly, such a simplified auditory environment with most of its sound energy at $7 \mathrm{kHz}$ could have led to a simplified, smaller A1. The greater vulnerability of lower tuned sites can possibly be explained by their inherently higher threshold, exaggerating further the activity discrepancy favoring $7 \mathrm{kHz}$ tuning in the cortex.

Rather surprisingly, pure tone exposure during the CP had no effect on A1 response thresholds, latencies, or tuning bandwidths. In rats undergoing exposures to different frequencies on different postnatal exposure schedules, Zhang et al. (2001) described a modest but significant BW10 increase for rats exposed. The immaturity before P20 of an intracortical inhibitory network capable of modulating tuning bandwidth in A1 (Kaur et al., 2004; Chang et al., 2005) could explain the lack of effect on BW20s. The stability of threshold and latency measurements despite CP exposure could reflect the reliance of these response properties on largely inflexible electromechanical properties of the peripheral auditory system and subcortical structures (Ehret and Romand, 1992).

Changes in the GABAergic system in the rat auditory cortex parallel changes in excitatory receptive fields, inhibitory receptive fields, and temporal response characteristics in A1 (Chang et al., 2005). It is therefore possible that the anesthetic used in this study influenced the estimation of threshold, bandwidth, and latency in younger animals. It is, however, unlikely that the anesthesia biased the main result of this study (CP window) because the exposure during early development was done on unanesthetized animals. Furthermore, the dependent variable measured to quantify the opening and closing of the $\mathrm{CP}$ is based on total A1 area size and RF CF, which, in subcortical nuclei and in the auditory cortex, tends not to be significantly influenced by the type of anesthetic used (Astl et al., 1996; Cheung et al., 2001).

\section{Impacts of exposure on the A1 representation of sound intensity}

Neurons with nonmonotonic rate/sound level functions play an essential role in sound intensity coding in the auditory system (Phillips and Irvine, 1981; Sutter and Schreiner, 1995), providing the brain with crucial information necessary for vocal communication and the perception of the distance separating an animal or human from other sound sources (Moore, 1995). Seven kilohertz $\mathrm{CP}$ exposure resulted in a significant increase in nonmonotonic discharge rate versus stimulus intensity functions for neurons recorded within A1, for both 7-kHz-tuned and all other A1 receptive fields (also see Zhang et al., 2001). Similar changes in "intensity functions" have been recorded in adult rats that have been behaviorally trained to detect sounds of a specific loudness (Polley et al., 2004, 2006), and in adult rats in which sound stimuli of a given intensity have been paired with pulsed acetylcholine release (Bao et al., 2004). Recent studies have shown that this behaviorally driven remodeling in older rats is accounted for by specific changes in cortical inhibitory input strengths that emerge at higher stimulus levels (Tan et al., 2006), consistent with the long-standing argument that nonmonotonic intensity functions are shaped by local (in A1, cortical) inhibition (Phillips et al., 1985). This striking sound exposure-induced effect suggests that simple pure-tone exposure during the $\mathrm{CP}$ induces inhibitory response remodeling in the cortical network itself. These current studies strongly indicate that there is some capacity for plasticity of inhibitory influences in the CP [as have been richly documented in V1 (Fagiolini and Hensch, 2000; Morales et al., 2002; Iwai et al., 2003; Hensch, 2005)] in these very young rats.

It might be noted that the "best levels" for nearly all nonmonotonic intensity functions generated in these exposed rats were for sound intensities at or below the intensity applied in the exposure environment (data not shown). This indicates that, as in the case of behaviorally trained animals (Polley et al., 2004, 2006), the specific sound intensities received by the rat pups in the exposure appeared to account for a selective change in inhibitory vs excitatory inputs that was specific to the applied stimulus intensities.

The almost equal extension of these effects to sectors of A1 representing non-7 kHz sound frequencies is of special interest because it implies that local stimulation can result in a distortion in the representation of sound intensity that applies for all simple acoustic inputs. Distortions in loudness representations are a common aspect of developmental impairments in language and cognition (Khalfa et al., 2004; Richardson et al., 2004). These results bear implications for understanding the origins of what can be general, severe perceptual distortions of loudness in these special child populations.

\section{Relationship of the CP window to auditory system development}

Tone-evoked responses were initially recorded in $\mathrm{A} 1$ in these rats at P11. As noted by Zhang et al. (2001), they were initially limited to the midfrequency sound range, and effectively excited only by relatively high-intensity stimuli. Over the subsequent $3 \mathrm{~d}$, extensive changes led to the emergence of simple, adult-like lowthreshold receptive fields, and to adult-like tonotopy. These findings are consistent with several studies that have documented a rapid maturation of the peripheral and central auditory system during the same developmental period in the rat. For example, the first auditory brainstem responses for air-conducted stimuli are obtained between P10 and P12 (Iwasa and Potsic, 1982; Blatchley et al., 1987; Geal-Dor et al., 1993). The cochlea undergoes its most dramatic postnatal changes between P12 and P14 (Puel and Uziel, 1987; Oliver and Fakler, 1999), and the tonotopic map within the medial nucleus of the trapezoid body in the pontine brainstem reaches its adult functional status by P14 (Friauf, 1992). At the cortical level, evoked potentials are first recordable at $\mathrm{P} 10$, and by $\mathrm{P} 14$, all of the components of an adultlike evoked response are present (Mourek et al., 1967). Metherate and Aramakis (1999) studied intrinsic properties of neurons in the rat auditory cortex and found that on-spiking and rectifying cells, which may promote the influence of transients in shaping cortical circuitry, grow progressively in numbers between P11 and P15. Finally, the end of the second week of life in the rat (P11 to P14) marks the emergence of the auditory orientation responses (Kelly et al., 1987). This behavioral change is paralleled by a rapid increase in the frequency and complexity of rat pup calls (Brudzynski et al., 1999).

Before P13, only high-intensity stimuli drive the immature auditory system. Using a normalized, louder sound stimulus at 
early dates to compensate for higher thresholds could potentially have led to an earlier estimated date for the opening of the CP. The shift would however have been of at most 1 or $2 \mathrm{~d}$ because before $\mathrm{P} 9$, even sounds of $110 \mathrm{~dB}$ cannot elicit auditory brainstem responses (Geal-Dor et al., 1993).

In our study, we found that receptive fields in A1 were sharply tuned to tonal stimuli at very young ages, with BW20s being narrower at $\mathrm{P} 11$ than at $\mathrm{P} 17$, when values appeared to plateau. This result is similar to findings in the developing cat cortex (Eggermont, 1996; Bonham et al., 2004; Pienkowski and Harrison, 2005), but differs from previous rat study descriptions (Zhang et al., 2001; Chang and Merzenich, 2003). This discrepancy in rats likely stems from differences in the sampled cortical area. More broadly tuned sites included in the latter studies would have been excluded in the present study as non-A1 sites.

\section{The further study of CP plasticity in the rat A1}

With this definition of the time of onset, duration, and closure of the CP, many additional studies of the powerful plasticity marking this early hearing epoch in the rat can be undertaken. This is an especially exploitable model because of the facility with which the exposure environment can be manipulated.

The short duration of the CP limited to the period of maturation of hearing in the rat raises interesting questions about its neurobiological significance for this rodent. In general, rat dams rear their litters in quiet, protected environments. Because the auditory system and cortex are subject to potential large-scale distortions over this epoch, that sequestering of pups in a quiet nest might be viewed as an ethological strategy for limiting any uncontrolled distortions in auditory representations that might confer later competitive disadvantages. A large proportion of the sounds in the nesting environment are generated by the movements and vocalizations of the dam and her pups, and there is a sharp increase in vocal productions during this early hearing (CP) period. There is a high likelihood that the specific environmental sounds associated with this epoch will be representationally exaggerated through CP plasticity. In that event, those early changes might facilitate the later communication and social interactions of conspecifics. Current studies are being conducted to determine if that is indeed the case.

\section{References}

Astl J, Popelar J, Kvasnak E, Syka J (1996) Comparison of response properties of neurons in the inferior colliculus of guinea pigs under different anesthetics. Audiology 35:335-345.

Bao S, Chang EF, Heiser MA, Merzenich MM (2003a) Representation of complex sounds through early experience. Soc Neurosci Abstr 29:182.16.

Bao S, Chang EF, Davis JD, Gobeske KT, Merzenich MM (2003b) Progressive degradation and subsequent refinement of acoustic representations in the adult auditory cortex. J Neurosci 23:10765-10775.

Bao S, Chang EF, Woods J, Merzenich MM (2004) Temporal plasticity in the primary auditory cortex induced by operant perceptual learning. Nat Neurosci 7:974-981.

Bartoletti A, Medini P, Berardi N, Maffei L (2004) Environmental enrichment prevents effects of dark-rearing in the rat visual cortex. Nat Neurosci 7:215-216.

Berardi N, Pizzorusso T, Maffei L (2000) Critical periods during sensory development. Curr Opin Neurobiol 10:138-145.

Berardi N, Pizzorusso T, Ratto GM, Maffei L (2003) Molecular basis of plasticity in the visual cortex. Trends Neurosci 26:369-378.

Blatchley BJ, Cooper WA, Coleman JR (1987) Development of auditory brainstem response to tone pip stimuli in the rat. Brain Res 429:75-84.

Bonham BH, Cheung SW, Godey B, Schreiner CE (2004) Spatial organization of frequency response areas and rate/level functions in the developing AI. J Neurophysiol 91:841-854.

Brudzynski SM, Kehoe P, Callahan M (1999) Sonographic structure of isolation-induced ultrasonic calls of rat pups. Dev Psychobiol 34:195-204.

Chang EF, Merzenich MM (2003) Environmental noise retards auditory cortical development. Science 300:498-502.

Chang EF, Bao S, Imaizumi K, Schreiner CE, Merzenich MM (2005) Development of spectral and temporal response selectivity in the auditory cortex. Proc Natl Acad Sci USA 102:16460-16465.

Cheung SW, Nagarajan SS, Bedenbaugh PH, Schreiner CE, Wang X, Wong A (2001) Auditory cortical neuron response differences under isoflurane versus pentobarbital anesthesia. Hear Res 156:115-127.

Clements M, Kelly JB (1978) Directional responses by kittens to an auditory stimulus. Dev Psychobiol 11:505-511.

Clopton BM, Winfield JA (1976) Effect of early exposure to patterned sound on unit activity in rat inferior colliculus. J Neurophysiol 39:1081-1089.

Daw NW (2005) Visual development, Ed 2. New York: Springer.

Diamond DM, Weinberger NM (1989) Role of context in the expression of learning-induced plasticity of single neurons in auditory cortex. Behav Neurosci 103:471-494.

Dorf DS, Curtin JW (1982) Early cleft palate repair and speech outcome. Plast Reconstr Surg 70:74-81.

Eggermont JJ (1996) Differential maturation rates for response parameters in cat primary auditory cortex. Aud Neurosci 2:309-327.

Ehret G, Romand R (1992) Development of tone response thresholds, latencies and tuning in the mouse inferior colliculus. Brain Res Dev Brain Res 67:317-326.

Fagiolini M, Hensch TK (2000) Inhibitory threshold for critical-period activation in primary visual cortex. Nature 404:183-186.

Fagiolini M, Pizzorusso T, Berardi N, Domenici L, Maffei L (1994) Functional postnatal development of the rat primary visual cortex and the role of visual experience: dark rearing and monocular deprivation. Vision Res 34:709-720.

Feller MB (1999) Spontaneous correlated activity in developing neural circuits. Neuron 22:653-656.

Friauf E (1992) Tonotopic order in the adult and developing auditory system of the rat as shown by c-fos immunocytochemistry. Eur J Neurosci 4:798-812.

Gao WJ, Wormington AB, Newman DE, Pallas SL (2000) Development of inhibitory circuitry in visual and auditory cortex of postnatal ferrets: immunocytochemical localization of calbindin- and parvalbumincontaining neurons. J Comp Neurol 422:140-157.

Geal-Dor M, Freeman S, Li G, Sohmer H (1993) Development of hearing in neonatal rats: air and bone conducted ABR thresholds. Hear Res 69:236-242.

Goodman J, Nusbaum HC (1994) The Development of speech perception: the transition from speech sounds to spoken words. Cambridge, MA: MIT.

Guillery RW, Stelzner DJ (1970) The differential effects of unilateral lid closure upon the monocular and binocular segments of the dorsal lateral geniculate nucleus in the cat J Comp Neurol 139:413-421.

Hanover JL, Huang ZJ, Tonegawa S, Stryker MP (1999) Brain-derived neurotrophic factor overexpression induces precocious critical period in mouse visual cortex. J Neurosci 19:RC40(1-5).

Harrison RV, Stanton SG, Ibrahim D, Nagasawa A, Mount RJ (1993) Neonatal cochlear hearing loss results in developmental abnormalities of the central auditory pathways. Acta Otolaryngol 113:296-302.

Hensch TK (2004) Critical period regulation. Annu Rev Neurosci 27:549-579.

Hensch TK (2005) Critical period mechanisms in developing visual cortex. Curr Top Dev Biol 69:215-237.

Iwai Y, Fagiolini M, Obata K, Hensch TK (2003) Rapid critical period induction by tonic inhibition in visual cortex. J Neurosci 23:6695-6702.

Iwasa H, Potsic WP (1982) Maturational change of early, middle, and late components of the auditory evoked responses in rats. Otolaryngol Head Neck Surg 90:95-102.

Kaur S, Lazar R, Metherate R (2004) Intracortical pathways determine breadth of subthreshold frequency receptive fields in primary auditory cortex. J Neurophysiol 91:2551-2567.

Kelly JB, Judge PW, Fraser IH (1987) Development of the auditory orientation response in the albino rat (Rattus norvegicus). J Comp Psychol 101:60-66.

Khalfa S, Bruneau N, Roge B, Georgieff N, Veuillet E, Adrien JL, Barthelemy 
C, Collet L (2004) Increased perception of loudness in autism. Hear Res 198:87-92.

Kilgard MP, Merzenich MM (1998) Cortical map reorganization enabled by nucleus basalis activity. Science 279:1714-1718.

King AJ, Hutchings ME, Moore DR, Blakemore C (1988) Developmental plasticity in the visual and auditory representations in the mammalian superior colliculus. Nature 332:73-76.

Knudsen EI (1998) Capacity for plasticity in the adult owl auditory system expanded by juvenile experience. Science 279:1531-1533.

LeVay S, Wiesel TN, Hubel DH (1980) The development of ocular dominance columns in normal and visually deprived monkeys. J Comp Neurol 191:1-51.

McGee AW, Yang Y, Fischer QS, Daw NW, Strittmater SM (2005) Experience-driven plasticity of visual cortex limited by myelin and Nogo receptor. Science 309:2222-2226.

Merzenich MM, Nelson RJ, Stryker MP, Cynader MS, Schoppmann A, Zook JM (1984) Somatosensory cortical map changes following digit amputation in adult monkeys. J Comp Neurol 224:591-605.

Metherate R, Aramakis VB (1999) Intrinsic electrophysiology of neurons in thalamorecipient layers of developing rat auditory cortex. Brain Res Dev Brain Res 115:131-144.

Moore BJ (1995) Hearing. New York: Academic.

Moore DR, Hutchings ME, Meyer SE (1991) Binaural masking level differences in children with a history of otitis media. Audiology 30:91-101.

Morales B, Choi SY, Kirkwood A (2002) Dark rearing alters the development of GABAergic transmission in visual cortex. J Neurosci 22:8084-8090.

Mourek J, Himwich WA, Myslivecek J, Callison DA (1967) The role of nutrition in the development of evoked cortical responses in rat, Brain Res 6:241-251.

Mower GD (1991) The effect of dark rearing on the time course of the critical period in cat visual cortex. Brain Res Dev Brain Res 58:151-158.

Nakahara H, Zhang LI, Merzenich MM (2004a) Specialization of primary auditory cortex processing by sound exposure in the "critical period." Proc Natl Acad Sci USA. 101:7170-7174.

Nakahara H, Zhang Houde JF, Schreiner CE, Merzenich MM (2004b) Changes in primary and secondary auditory cortex by exposure to the phonemes. Soc Neurosci Abstr 30:752.3.

Oliver D, Fakler B (1999) Expression density and functional characteristics of the outer hair cell motor protein are regulated during postnatal development in rat, J Physiol 519:791-800.

Phillips DP, Irvine DR (1981) Responses of single neurons in physiologically defined primary auditory cortex (AI) of the cat: frequency tuning and responses to intensity. J Neurophysiol 45:48-58.

Phillips DP, Orman SS, Musicant AD, Wilson GF (1985) Neurons in the cat's primary auditory cortex distinguished by their responses to tones and wide-spectrum noise. Hear Res 18:73-86.

Pienkowski M, Harrison RV (2005) Tone responses in core versus belt auditory cortex in the developing chinchilla. J Comp Neurol 7:101-109.

Pizzorusso T, Medini P, Berardi N, Chierzi S, Fawcett JW, Maffei L (2002) Reactivation of ocular dominance plasticity in the adult visual cortex. Science 298:1248-1251.

Pizzorusso T, Medini P, Landi S, Baldini S, Berardi N, Maffei L (2006) Structural and functional recovery from early monocular deprivation in adult rats. Proc Natl Acad Sci USA 103:8517-8522.

Polley DB, Steinberg EE, Merzenich MM (2006) Perceptual learning directs auditory cortical map reorganization through top-down influences. J Neurosci 26:4970-4982.

Polley DB, Heiser MA, Blake DT, Schreiner CE, Merzenich MM (2004) Associative learning shapes the neural code for stimulus magnitude in primary auditory cortex. Proc Natl Acad Sci USA 101:16351-16356.

Poon PW, Chen X (1992) Postnatal exposure to tones alters the tuning characteristics of inferior collicular neurons in the rat. Brain Res 585:391-394.

Puel JL, Uziel A (1987) Correlative development of cochlear action potential sensitivity, latency, and frequency selectivity, Brain Res 465:179-188.

Rajan R, Irvine DR, Wise LZ, Heil P (1993) Effect of unilateral partial cochlear lesions in adult cats on the representation of lesioned and unlesioned cochleas in primary auditory cortex. J Comp Neurol 338:17-49.
Ramachandran R, Davis KA, May BJ (1999) Single-unit responses in the inferior colliculus of decerebrate cats. I. Classification based on frequency response maps. J Neurophysiol 82:152-163.

Rauschecker JP (1998) Cortical processing of complex sounds. Curr Opin Neurobiol 8:516-521.

Rauschecker JP (1999) Auditory cortical plasticity: a comparison with other sensory systems. Trends Neurosci 22:74-80.

Recanzone GH, Jenkins WM, Hradek GT, Merzenich MM (1992) Progressive improvement in discriminative abilities in adult owl monkeys performing a tactile frequency discrimination task. J Neurophysiol 67:1015-1030.

Rice FL, Van der Loos H (1977) Development of the barrels and barrel field in the somatosensory cortex of the mouse. J Comp Neurol 171:545-560.

Richardson U, Thomson JM, Scott SK, Goswami U (2004) Auditory processing skills and phonological representation in dyslexic children. Dyslexia 10:215-233.

Richman LC, Eliason MJ, Lindgren SD (1988) Reading disability in children with clefts. Cleft Palate J 25:21-25.

Sanes DH, Constantine-Paton M (1983) Altered activity patterns during development reduce neural tuning. Science 221:1183-1185.

Sanes DH, Constantine-Paton M (1985) The sharpening of frequency tuning curves requires patterned activity during development in the mouse, Mus musculus. J Neurosci 5:1152-1166.

Schlaggar BL, O’Leary DD (1993) Patterning of the barrel field in somatosensory cortex with implications for the specification of neocortical areas. Perspect Dev Neurobiol 1:81-91.

Schoups A, Vogels R, Qian N, Orban G (2001) Practising orientation identification improves orientation coding in V1 neurons. Nature 412:549-553.

Shatz CJ (1990) Impulse activity and the patterning of connections during CNS development. Neuron 5:745-756.

Shatz CJ, Stryker MP (1978) Ocular dominance in layer IV of the cat's visual cortex and the effects of monocular deprivation J Physiol 281:267-283.

Simons DJ, Land PW (1987) Early experience of tactile stimulation influences organization of somatic sensory cortex. Nature 326:694-697.

Stern EA, Maravall M, Svoboda K (2001) Rapid development and plasticity of layer 2/3 maps in rat barrel cortex in vivo. Neuron 31:305-315.

Sutter ML, Schreiner CE (1995) Topography of intensity tuning in cat primary auditory cortex: single-neuron versus multiple-neuron recordings. J Neurophysiol 73:190-204.

Taha SA, Stryker MP (2005) Molecular substrates of plasticity in the developing visual cortex. Prog Brain Res 147:103-114.

Tan AY, Atencio CA, Polley DB, Merzenich MM, Schreiner CE (2006) Unbalanced synaptic inhibition can create intensity-tuned auditory cortex neurons. arXiv:q-bio.NC/0607036/.

Wiesel TN, Hubel DH (1963) Single-cell responses in striate cortex of kittens deprived of vision in one eye. J Neurophysiol 26:1003-1017.

Wiesel TN, Hubel DH (1965) Comparison of the effects of unilateral and bilateral eye closure on cortical unit responses in kittens. J Neurophysiol 28:1029-1040.

Xerri C, Coq JO, Merzenich MM, Jenkins WM (1996) Experience-induced plasticity of cutaneous maps in the primary somatosensory cortex of adult monkeys and rats. J Physiol Paris 90:277-287.

Yan J, Suga N (1996) Corticofugal modulation of time-domain processing of biosonar information in bats. Science 273:1100-1103.

Yan W, Suga N (1998) Corticofugal modulation of the midbrain frequency map in the bat auditory system. Nat Neurosci 1:54-58.

Zhang LI, Bao S, Merzenich MM (2001) Persistent and specific influences of early acoustic environments on primary auditory cortex. Nat Neurosci 4:1123-1130.

Zhang LI, Bao S, Merzenich MM (2002) Disruption of primary auditory cortex by synchronous auditory inputs during a critical period. Proc Natl Acad Sci USA 99:2309-2314

Zhang Y, Suga N (1997) Corticofugal amplification of subcortical responses to single tone stimuli in the mustached bat. J Neurophysiol 78:34893492. 